\title{
Acoustic Emission for Evaluating the Performance of Steel Fiber Reinforced Concrete ${ }^{\dagger}$
}

\author{
Anastasios Mpalaskas ${ }^{1}$, Dimitrios Aggelis ${ }^{2, *}$ and Ninel Alver ${ }^{3}$ \\ 1 Department of Materials Science and Engineering, University of Ioannina, Ioannina 45110, Greece; \\ tbalask@gmail.com \\ 2 Department Mechanics of Materials and Constructions, Vrije Universiteit Brussel (VUB), Pleinlaan 2, 1050 \\ Brussels, Belgium \\ 3 Department of Civil Engineering, Ege University, Bornova, Izmir 35040, Turkey; ninel.alver@gmail.com \\ * Correspondence: Dimitrios.Aggelis@vub.be \\ † Presented at 1st International Electronic Conference on Applied Sciences, 10-30 November 2020; Available \\ online: https://asec2020.sciforum.net/.
}

Published: 10 November 2020

\begin{abstract}
Steel fiber reinforcement in concrete strongly enhances its ductility and toughness. This is basically due to the additional fracture mechanisms and energy to overcome the interlocking and adhesion between the fibers and the cementitious matrix. The enhancement of the final properties is measured by mechanical tests and can be assessed only at the end of loading. These processes can be well monitored by acoustic emission (AE) indices offering real-time characterization of the material's performance much earlier than the final failure or the termination of loading. In this study, steel fiber reinforced concrete (SFRC) beams were tested in bending with simultaneous AE monitoring. Tests conducted independently in different laboratories confirm that the AE behavior at low load levels is very indicative of the amount of reinforcement and consequently, of the final mechanical properties. The reason is that the reinforcement phase is activated through shear stresses in the interphase, a mechanism that is more profound in the presence of higher fiber content, and correspondingly is absent in plain unreinforced material. This finding opens the way to characterize the effectiveness of reinforcement with just a proof loading at less than $20 \%$ of the final load bearing capacity.
\end{abstract}

Keywords: AE parameters, bending, concrete, RA-value, duration, interphase, steel fibers

Publisher's Note: MDPI stays neutral with regard to jurisdictional claims in published maps and institutional affiliations.

(C) 2020 by the authors. Submitted for possible open access publication under the terms and conditions of the Creative Commons Attribution (CC BY) license (http://creativecommons.org/licenses/by/4.0/). 